Canadian University Music Review

Revue de musique des universités canadiennes

\title{
Le temps musical
}

\section{Serge Garant}

\author{
Numéro 7, 1986
}

URI : https://id.erudit.org/iderudit/1014085ar

DOI : https://doi.org/10.7202/1014085ar

Aller au sommaire du numéro

\section{Éditeur(s)}

Canadian University Music Society / Société de musique des universités

canadiennes

\section{ISSN}

0710-0353 (imprimé)

2291-2436 (numérique)

Découvrir la revue

\section{Citer ce document}

Garant, S. (1986). Le temps musical. Canadian University Music Review / Revue de musique des universités canadiennes, (7), 32-35.

https://doi.org/10.7202/1014085ar

All Rights Reserved (c Canadian University Music Society / Société de musique des universités canadiennes, 1986
Ce document est protégé par la loi sur le droit d'auteur. L’utilisation des services d'Érudit (y compris la reproduction) est assujettie à sa politique d'utilisation que vous pouvez consulter en ligne.

https://apropos.erudit.org/fr/usagers/politique-dutilisation/ 


\title{
LE TEMPS MUSICAL
}

\author{
Serge Garant
}

Le texte que voici est tiré de l'ultime entrevue accordée publiquement par Garant. Recueilli par Marie-Thérèse Lefebvre le 17 juillet 1986, l'entretien a été publié intégralement dans l'ouvrage que cette dernière a consacré au compositeur (1986 : 236-239). Le fragment ici retenu est particulièrement intéressant en ce qu'il fait entendre certaines préoccupations méconnues de Garant relativement à une dimension fondamentale de la musique : la temporalité.

Ce qui me préoccupe d'abord, c'est la perception du temps : le fait qu'une oeuvre de Webern qui est effectivement courte puisse sembler longue parce que complexe, alors que des oeuvres longues sont justement parfois trop longues parce qu'il n'arrive rien. La perception qu'on a d'une oeuvre varie énormément selon la qualité du temps et non sa quantité. La densité de certains événements, par exemple chez Boulez, la rapidité même des événements fait que, dans un sens, le détail ne compte plus et pourtant l'oeuvre est travaillée de façon extraordinaire et les coloris y sont dessinés avec une finesse incroyable. Seulement, les événements sont tellement rapides, il y a tellement d'informations données en peu de temps, que ce que l'on perçoit, c'est une ligne générale. On retient un départ, puis une arrivée. On ne retrouve pas ce phénomène chez Webern où, au contraire, on èst presque toujours pris par les détails. Il y a des exceptions tout de même : dans les mouvements extrêmement polyphoniques et contrapuntiques, comme le dernier mouvement de la dernière Cantate, on retient une ligne générale car l'oreille ne peut capter le détail de chacune des lignes. [...] 
Chez Boulez, même si le tempo est lent, ce n'est toujours qu'une suspension dans le mouvement car on repart immédiatement. Ces mouvements lents me semblent être une interruption d'une matière rapide plutôt qu'une chose en soi. Ce ne sont pas les silences qui produisent cet effet mais la miniaturisation des événements qui, chez Webern, sont une chose en soi. C'est donc une question de conception de la matière globale et le problème du compositeur est de conserver une perception du temps réel pendant qu'il compose. Cela n'est possible que s'il parvient à prendre ses distances avec l'oeuvre en gestation. Il est possible que Webern n'ait pas eu cette conscience ou encore que ce soit un problème d'édition. Il est aussi possible que Webern ne se soit pas occupé de la durée réelle mais qu'il ait simplement préféré jouer avec les structures, celles-ci étant d'une telle complexité qu'elles donnent l'impression que l'oeuvre durera longtemps.

Dans ma démarche de compositeur, je me préoccupe beaucoup de ce phénomène de perception du temps réel d'une oeuvre. J'essaie de prendre mes distances, de regarder mon papier et de chanter en temps réel afin de prendre conscience de la durée d'un événement. [...] Il y a des oeuvres très rapides qui donnent toute leur information dans les 10 premières mesures et après, ne font que "brasser la soupe" : tout est dit. Alors qu'une oeuvre comme la Suite lyrique [de Berg] est si riche de matière qu'elle donne l'impression de durer.

Il y a deux types de mouvements rapides ou même de mouvements lents. Un mouvement lent construit sur la forme A B A donne une impression de redite alors qu'un mouvement lent qui s'invente une musique constamment doit être écouté avec beaucoup plus d'attention, la matière y étant plus intense. La forme colore ainsi la durée de la musique.

Le temps musical est donc un phénomène complexe qui exigerait une réflexion plus approfondie. Et on peut se demander si c'est seulement ce qui est analysable qui s'imprime dans notre cerveau. On peut aussi se demander s'il n'y a pas tout un aspect du découpage temporel qu'on ne peut analyser. J'ai construit longtemps des formes qui reposaient 
sûr cette idée, c'est-à-dire que les proportions temporelles de l'oeuvre étaient issues de celles inscrites dans la cellule initiale. Je ne prétends pas que l'auditeur puisse se rendre compte de ces rapports, mais je prétends qu'inconsciemment, un équilibre s'impose entre la matière fine et la matière globale. Ce découpage formel est le résultat de celui des hauteurs, mais tout ceci est purement abstrait car la qualité des événements n'est pas décidée a priori. Il en résulte donc que les séquences d'une telle durée quantitative peuvent sembler qualitativement autres.

Dans la musique répétitive, par contre, le temps est traité par paliers, comme un escalier. On monte une marche et on attend qu'un événement nous fasse monter une autre marche. Mais la perception du temps dans un autre type de musique est différente. On n'y attend pas un élément déclencheur. La musique répétitive ressemble à une série de salles qu'on visite alors que la perception dont je parle est une promenade dans un long corridor. On va plus ou moins vite, selon ce que l'on voit ou pour toute autre raison. Ou encore, on peut penser à une sorte de pente en hélice que l'on peut monter à la vitesse que l'on veut. Il n'y a pas un événement précis qui nous oblige à changer de palier. Il est vrai cependant que dans la musique répétitive, on ne change pas nécessairement de façon abrupte. Il arrive que l'on change si lentement que c'est "la goutte qui fait déborder le vase" qui provoque le changement : et on ne se rend pas compte de ce changement, on le subit.

Il y a aussi des musiques où le temps est vraiment suspendu, comme le début de l'Adagio de l'Opus 106, de Beethoven, cette page absolument extraordinaire, d'une telle profondeur et d'une telle audace dans la nudité que le temps n'existe plus. La façon dont s'y prend le compositeur pour créer un tel monde sonore ne relève pas d'une question de technique : c'est une question spirituelle. On peut suspendre le temps autant dans la vitesse (Boulez le fait dans certaines oeuvres où les événements sont tellement fulgurants que le découpage formel disparaît, on n'y pense plus) que dans la lenteur, comme dans cette oeuvre de Beethoven 
où ce n'est pas "autre chose" qui arrive mais l'inverse. Il y a une telle respiration profonde que le temps n'existe plus. Bien sûr, techniquement, il existe des façons de suspendre le temps : utiliser des registres fixes par exemple, mais cela n'a pas beaucoup d'intérêt, car c'est "un truc", et ce qui m'intéresse ce n'est pas "le truc", mais le fait d'être constamment au coeur de l'action. [...]

Il y a une part de déterminisme dans ma musique mais aussi une part de gratuité. J'avais remarqué ce geste de gratuité chez un peintre, Francis Bacon, dont les tableaux donnent l'impression qu'à un certain moment, ils ont été effacés, à cause des distorsions du visage. On dirait que le peintre a d'abord peint un portrait très classique et qu'en donnant un coup d'éponge tout devint distordu. J'ai trouvé cette idée intéressante et $\mathrm{j}$ 'ai tenté la même chose en musique dans ...chant d'amours. Après avoir tout écrit selon des structures de départ, $j$ 'ai créé des vides en effaçant certains endroits. En un sens, il reste des "ruines", mais ce sont les "bonnes ruines". Je n'ai pas répété ce geste par la suite, car il n'obéit pas à une méthode très rigoureuse. Ce qui m'amusait, c'était de garder le début d'une durée, la fin d'une autre, si bien qu'on ne sait plus où se fait la liaison entre les deux. Il y a dans ce geste quelque chose de gratuit qui fait partie de la composition.

\section{RÉFÉRENCE}

LEFEBVRE, M.T.

1986: Serge Garant et la révolution musicale au Québec. Montréal : Louise Courteau éditrice. 